\title{
BENDING FAILURE BEHAVIOR OF THE GLASS FIBER REINFORCED COMPOSITE I-BEAMS FORMED BY A NOVEL BENDING PULTRUSION PROCESSING TECHNIQUE
}

\author{
Lvtao Zhu', ${ }^{1,2}$ Shengbin $\mathrm{CaO}^{3, *}$, Xiaofeng Zhang ${ }^{4}$, Lei $\mathrm{Li}^{3}$ \\ 1 College of Textile Science and Engineering, Zhejiang Sci-Tech University, Zhejiang, People's Republic of China \\ 2 The Opening Project of Key Laboratory of Clean Dyeing and Finishing Technology of Zhejiang Province, Zhejiang, People's Republic of China \\ 3 School of Materials Science, Shanghai Dianji University, Shanghai, People's Republic of China \\ 4 School of Mechanical Engineering, Shanghai Dianji University, Shanghai, People's Republic of China \\ ${ }^{*}$ Corresponding author. E-mail: caosb@sdju.edu.cn
}

\begin{abstract}
:
The glass fiber reinforced resin matrix composite l-beams were designed and formed via a type of novel bending pultrusion processing technique, and the three-point bending tests were carried out to analyze the mechanical bending performances. The obtained results show that the main failure mode of the composite I-beam under the bending load is the upper structure (top flange) cracks along the length direction of the fibers, and the cracks simultaneously propagate downwards in the vertical direction. The bifurcated cracks can be found at the junction area between the top flange and web. In addition, the main bending failure mechanism of the composite I-beam includes the matrix cracking, propagation of cracks, and final fracture failure. In particular, noting that when the crack reaches the l-shaped neck position, the lateral bifurcation occurs, and the resulting secondary cracks further extend in two directions, which leads to the serious damage between the top flange and web, and the ultimate fracture failure occurs.
\end{abstract}

\section{Keywords:}

Bending pultrusion forming, glass fiber, three-point bending, failure mechanism

\section{Introduction}

The composite materials are composed of two or more different substances combined via the specific processing methods. The constituent substances are mainly divided into two types, namely the reinforcement and matrix. Among them, the reinforcement serves as the main supporting framework of the entire composite structure, i.e., the main load-bearing part. The matrix has the functions of transferring load and protecting the reinforcement, both of which contribute to the mechanical properties. Therefore, the composites can overcome the shortcomings of a single material, highlighting the advantages of each component, thus strengthening the mechanical performances, and expanding the applications of the composite structural parts [1]. In addition, these types of materials have many excellent properties such as high specific modulus, low specific gravity, high strength, and good design-ability [2-6]. They have been widely used in aviation, aerospace, transportation, civil engineering, protection, and other fields [7-9].

Based on the different types of matrix, there are mainly three types of composite materials: polymer-based, metal-based, and inorganic non-metal-based. Among them, metal-based and inorganic non-metal-based composite materials are more expensive and mainly used in military and aerospace. The processing technology of the polymer-based composites is more mature and has been widely used in industry [10, 11]. For reinforcing materials, high-performance fibers, such as glass fiber [12], carbon fiber [13], basalt fiber [14], and aramid fiber [15], with excellent mechanical properties are commonly used. Among them, glass fiber is a kind of inorganic non-metallic material with excellent performances, mainly containing silica, alumina, magnesia, calcium oxide, sodium oxide, and others, and it has the good chemical stability. For polymer matrix composites, resin is often used as the matrix. Therefore, the composite materials that use the resin and glass fibers as the matrix and reinforcement have a series of advantages such as light weight, high strength, heat resistance, corrosion resistance, and electrical insulation [16].

Further, the processing techniques such as vacuum-assisted liquid molding, compression molding, resin transfer molding, wet winding molding, and autoclave molding can be used to obtain the fiber-reinforced composite materials with the specific properties. In recent years, with the continuous progress of the manufacturing technologies, some relatively new technologies have emerged, such as the composite material additive manufacturing technology [17] and the high-performance bending pultrusion processing technique mentioned in this paper.

The bending pultrusion processing is a technique for obtaining composite products from fibers or fabrics under external force, through resin dipping, pultrusion molding, heating curing, winding, or cutting. It can be used for continuous production of composite products with the fixed cross-sectional shapes. This method uses a dehumidifying device to remove the 
moisture from the fiber before dipping and molding, thereby effectively reducing the moisture content and improving the quality of the products. The gluing process adopts closed injection. This process is different from the traditional open dipping. Simultaneously, it combines with the vacuum-assisted process to effectively reduce the porosity of the products. The traditional preforming, forming, dipping the glue, and other processes are unified in a mold, which ingeniously improves the preparation efficiency and reduces the waste of raw materials and environmental pollution. The outer shell of the comprehensive reaction mold is set according to the curvature of the internal curved channel, thereby saving the preparation mold, reducing the cost and the volume of the mold.

The structures of fiber-reinforced composite materials can be divided into I-beams, U-shaped beams, T-shaped beams, hatshaped beams, etc. According to the different cross-sectional shapes, the I-beams are one of the most widely studied and applied composites. Mohamad et al. [18] designed the composite I-beams in large wind turbine blades and studied the structure and performance optimization. Qin et al. [19, 20] developed an analysis solution suitable for horizontally curved composite I-beams. This solution is mainly used to solve the static problem of double-layer composite curved beams with flexible shear connections in the tangential direction. Lacki et al. [21] studied the effect of glass fiber content on the bending resistance of composite I-beam. By adding glass fiber content, the transverse torsional buckling of the I-beam web was reduced and the stiffness was improved. Gagan et al. [22] compared the bending behavior of glass fiber-reinforced polymer bonded I-beams and pultruded I-beams, and it showed that even if there are fiber interruptions and breaks at the junction of the wing and web, the performance change was not significant. In addition, Shohreh Alaedini et al. [23] used four-point bending to study the mechanical behavior of fiber-reinforced polymer fully pultruded I-beams and incomplete pultruded I-beams, and the impact of initial imperfections on the flexural torsional buckling capacity. Shinokubo et al. [24] designed several I-shaped woven fabrics mixed with glass fibers and carbon fibers and used them to strengthen composite I-beams. The four-point bending tests were used. By optimizing the fiber content, the bending strength can be improved. Zhu et al. [25] modified the composite I-beams with prestressed carbon fiber reinforced ribs to improve the bending rigidity. The four-point bending experiments revealed that it can greatly improve the mechanical performances of the I-beam. Based on the abovementioned descriptions, it can be found that the more detailed information on the mechanical behavior of the fiber reinforced composite I-beams needs to be presented.

In this paper, the three-point bending behavior of the glass fiber reinforced resin matrix composite l-beams formed by the presented novel bending pultrusion processing technique is experimentally studied. The bending failure mechanical performance and failure mode are analyzed. In particular, the bending failure mechanism is explored to provide the effective guidance for the performance optimization of the composite I-beams.

\section{Experimental}

\subsection{Materials}

The applied material is a kind of I-shaped cross-section fiberreinforced composite bending pultrusion profiles. Epoxy resin is selected as the matrix, and the reinforcement is made of glass fibers. Moreover, the glass fibers are arranged axially in parallel inside the profile.

\subsection{Material processing technology}

The composite materials were prepared by bending pultrusion molding process. As shown in Figure 1, the yarns on the creels 1 and 2 pass through the yarn guide, the dehumidifying device, the roller, the comprehensive reaction mold, the traction device, and the cutting device in sequence, and can be processed into the bending pultruded composite I-beam.

The entire preparation process was completed in an open pultrusion machine. First, each fiber was controlled by the tension creel, the tensile load was controlled to $0.5 \mathrm{kN}$, and the yarns were released at an uniform speed. Second, after the fibers were led out from the creels, they entered the dehumidification device to reduce the moisture content, and the dehumidification temperature was controlled at $60^{\circ} \mathrm{C}$. The dehumidified fibers passed through the threading device and entered the dipping tank. After the fibers were fully infiltrated in the dipping tank, they entered the pultrusion mold. After that, the mixture of fibers and resin entered the preheating zone $\left(125^{\circ} \mathrm{C}\right)$ for heating, then entered the gel zone $\left(165^{\circ} \mathrm{C}\right)$, where the resin began to undergo an exothermic reaction, changing from a viscous state to a gel state; then it entered the curing zone $\left(180^{\circ} \mathrm{C}\right)$, where the resin got cured and shrank. Because of the release agent, the solid mixture of fibers and resin no longer adhered to the inside of the mold. Finally, the traction device pulled the cured profile out and cut it into testing pieces, as shown in Figure 2. For the composite I-beam testing specimens, the fiber volume content was approximately $55 \%$. Besides, the number of testing specimens was 5 , and the bending performance tests were performed.

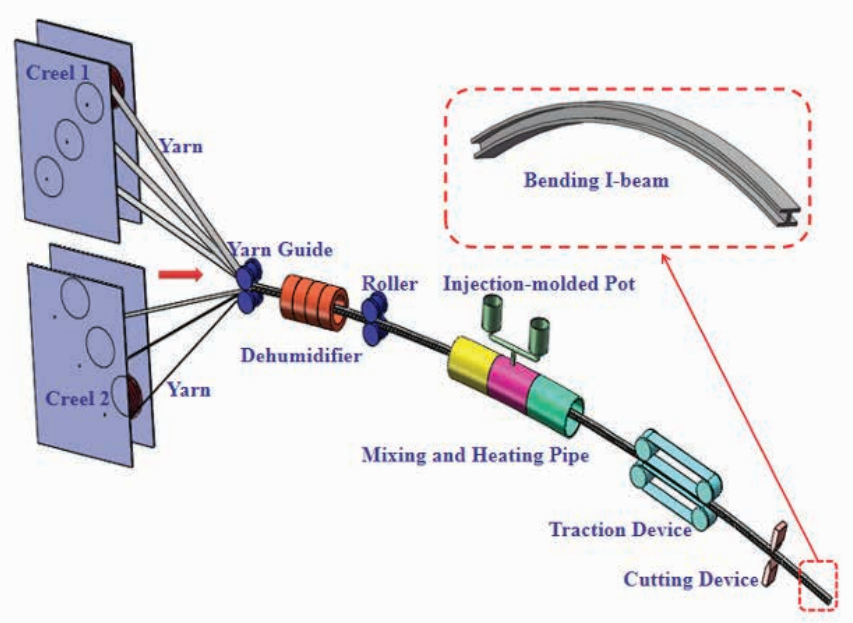

Figure 1. The bending pultrusion processing technique. 
(a)

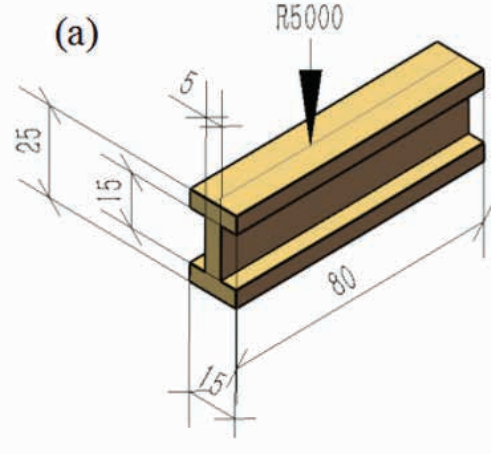

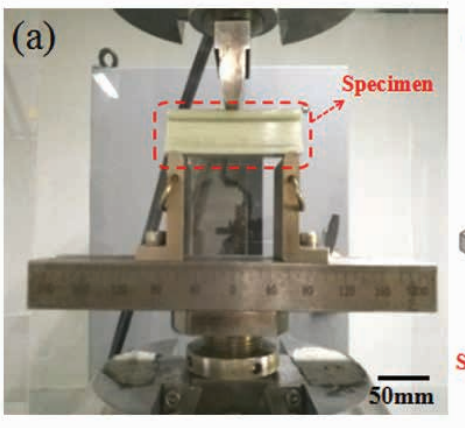

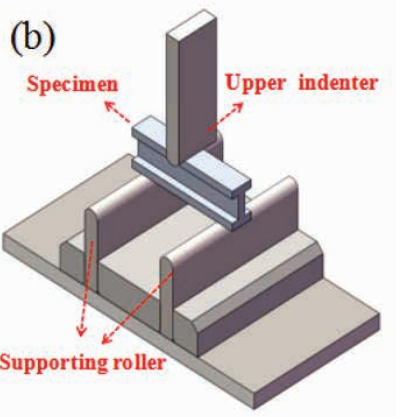

Figure 2. The glass fiber reinforced composite I-beam specimen (A) sizes of the I-beam ( $\mathrm{mm})$, (B) I-shaped cross-section, and (C) front view of the l-beam.

\subsection{Three-point bending tests}

According to the testing standard of GBT 13096-2008, the INSTRON ${ }^{\circledR}-8801$ mechanical tester was used to perform the three-point bending tests, as shown in Figure 3. Furthermore, the upper loading indenter was set to contact with the upper surface of the testing specimen. There were two supporting points on the lower surface of the specimen with a span of $66 \mathrm{~mm}$, which were symmetrical with each other, and the loading indenter's speed was $10 \mathrm{~mm} / \mathrm{min}$. When measuring the bending strength of the specimen, the load was applied continuously until the composite was damaged and could no longer bear the applied load, and then the test was terminated.

Further, to explore the failure mode of the damaged composite I-beams, the ZEISS Xradia610/Versa610 X-ray threedimensional microscope was used.

\section{Results and discussions}

\subsection{Bending stress-deflection curves}

During the three-point bending testing process, as the indenter moves slowly downwards at a uniform speed, the composite specimen will change mainly in two aspects. On the one hand, it is manifested in the externally visible deformation, i.e., the accumulation of deformation; on the other hand, it is manifested in the internal stress, i.e., the change of stress reflects the status of structural failure. Figure 4 shows the bending stressdeflection curves.

By investigating the stress status shown in Figure 4, it can be found that the stress gradually linearly increases after the specimen is bent and deformed under compression. As the deformation gradually increases during the loading process, a larger load is needed for the specimen to generate the continuous bending deformation. In particular, when the structural stress reaches the failure stress threshold of the resin matrix, a small yield occurs. At this moment, the structural failure has begun to occur. It is manifested in the corresponding curves that the stress has a buffer zone and the rate of increase slows down. However, the stress is still gradually increasing. The highest value occurs when the stress reaches the failure threshold of the fibers. After that, the regional failure of resin

Figure 3. Three-point bending testing (A) experimental setup and (B) schematic diagram.

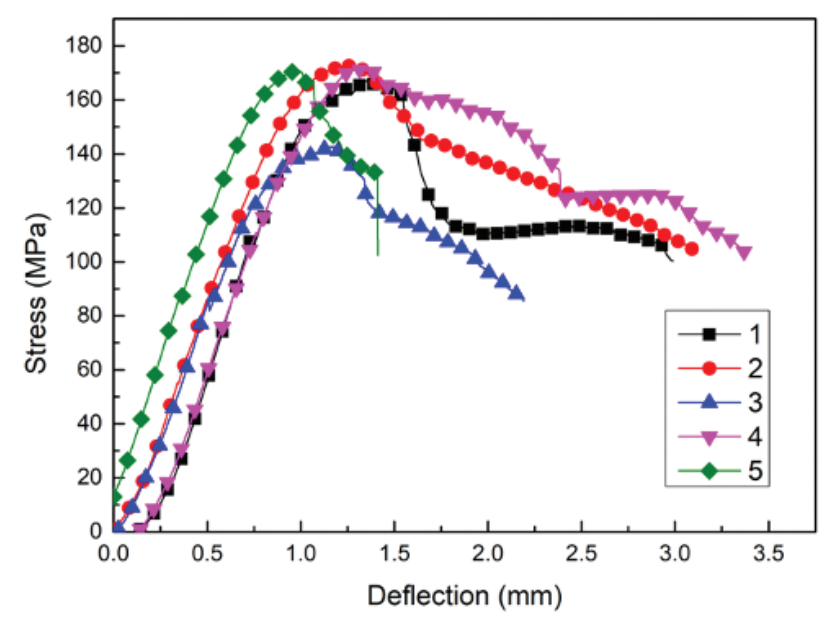

Figure 4. Bending stress-deflection curves.

and fibers continued to occur, which is manifested as small amplitude oscillations in stress. At this moment, the composite had failed and could no longer bear external loads.

\subsection{Damage mode}

By observing the failure morphologies of the tested composite I-beam specimens, it is found that the specimens have the similar fracture failure mode, as shown in Figure 5 . Besides, it indicates that the central part of the upper layer (top flange) of the testing specimen is significantly damaged due to the direct load bearing. The cracks mainly extend vertically downwards from the direct loading point and simultaneously propagate along the length direction of the upper surface of the specimen, i.e., expanding along the length direction of fibers to the end face.

Moreover, to further explore the specific failure mode of the composite T-beam subjected to three-point bending load, Figure 6 shows the typical final failure mode and the microscopic images. Note that when the vertically downward cracks reach the I-shaped neck position, i.e., the junction area between the top flange and web, the lateral bifurcation occurs, and the resulting secondary cracks further propagate in two directions, and thus the fracture failure occurs.

Therefore, the typical failure mode of the composite I-beam subjected to the continuous bending stress is that the upper 


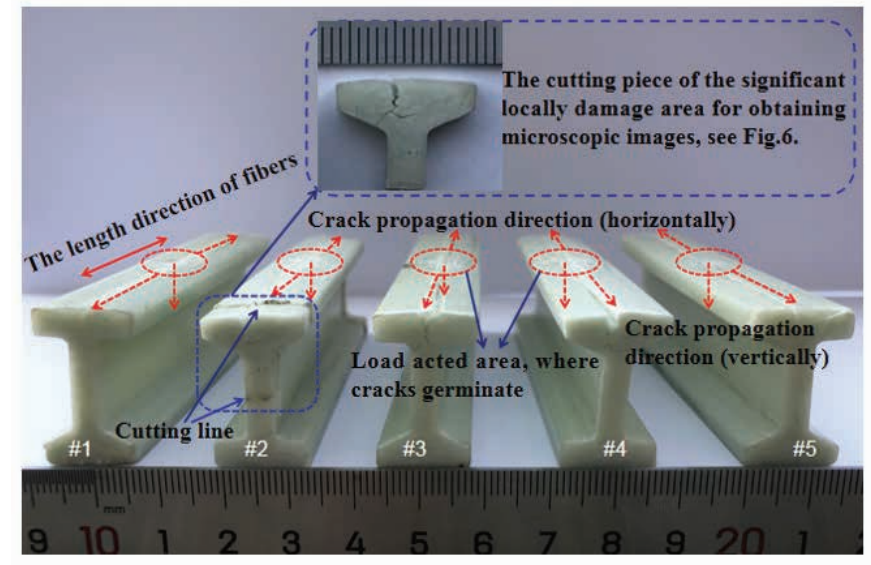

Figure 5. The failure morphologies of the tested composite I-beam specimens.

layer cracks mainly propagate along the length direction of fibers and simultaneously propagate downward along the vertical direction. Moreover, the bifurcation cracks occur at the junction area between the top flange and web, and the structure of bottom flange is slightly damaged. The reason for the occurrence of this phenomenon is closely related to the arrangement of fibers in the specimen, i.e., there is no binding effect of fibers through the thickness direction between the abovementioned three parts (top flange, web, and bottom flange), which causes the cracks to easily germinate and propagate.

\subsection{Damage mechanisms}

As mentioned above, the structure of the composite I-beam is obviously damaged along the load direction under the threepoint bending loading condition. The main failure modes are the cracking along the length direction of the fibers and the vertical direction from the top flange to the web.

The main failure mechanisms of the glass fiber resin matrix composite I-beam under three-point bending loading are cracking germination, cracking propagation, and the final fracture. First of all, during the loading process, as the failure stress of the matrix is significantly smaller than that of the glass fiber, when the matrix at the direct loading part reaches the stress threshold, the formation of matrix micro-cracks indicates the beginning of the initial stage of progressive damage. Then, as the composite is further stressed, the cracks propagate in the internal structure of the composite, i.e., the cracks further propagate along the length direction of fibers as well as the vertical direction. Finally, when the downwardly extending vertical cracks reach the junction area between the top flange and web, the lateral bifurcation occurs, and the resulting secondary cracks further propagate in two directions. Thus the ultimate fracture failure of the composite I-beam will occur in a short period of time.

\subsection{The suggestions for improving bending resistance performance}

Under bending load, the resin cracks and fiber fractures mainly appear on the top flange of the composite I-beam. Because the

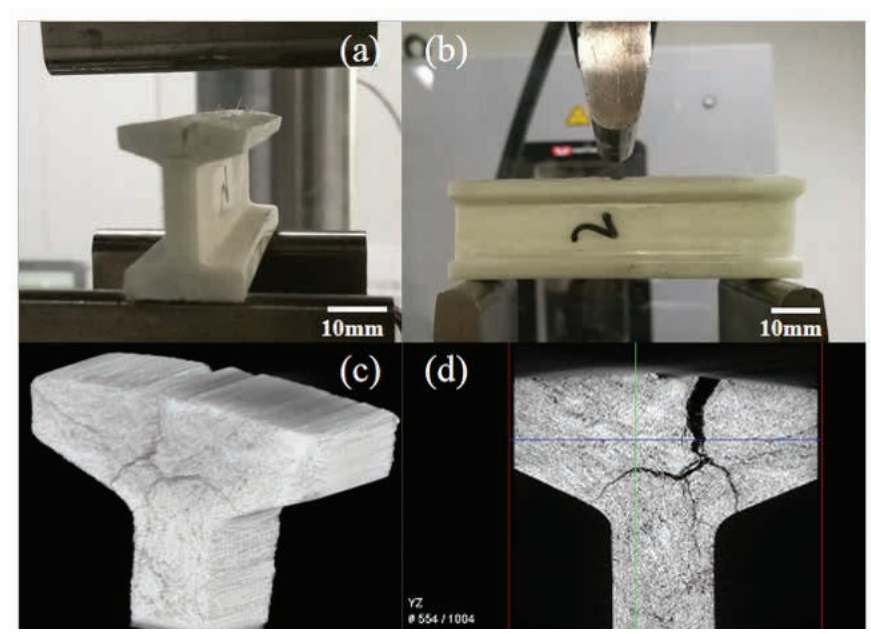

Figure 6. The typical failure morphology of the damaged composite I-beam. (A) and (B) the damaged specimen \#2, (C) and (D) microscopic images of the significant locally damage at the junction area between the top flange and web (specimen \#2).

fibers are distributed at $0^{\circ}$, the cracks propagate along the fiber direction to form axial cracks, while the cracks also propagate downward to generate longitudinal cracks. Furthermore, the cracks propagate in the junction area of top flange and web, forming multiple main cracks to expand separately until the ultimate fracture failure occurs.

Generally speaking, the top flange is the key area for the bending-induced damage of composite I-beam, whereas there is less visible damage on the web and bottom flange. When designing the composite I-beams to resist three-point bending loads, in addition to selecting the high-performance fibers and resin materials with excellent mechanical properties, the structural optimization for flanges should also be well considered. For example, the three-dimensional fabrics can be used as the reinforcing phase. This proposed structure can effectively prevent the generation and further propagation of cracks. In addition, the junction area between the top flange and web can be locally strengthened to avoid or slow down the propagation of lateral cracks.

\section{CONCLUSIONS}

In this paper, the glass fiber reinforced resin matrix composite I-beams were designed and manufactured via a type of novel bending pultrusion processing technique, and the threepoint bending tests were performed to analyze the bending performance, damage mode, and the failure mechanisms. The main conclusions are as follows:

1. During the three-point bending process, the composite I-beam mainly changes in two aspects. On the one hand, it is manifested in the externally visible deformation. On the other hand, the change of stress reflects the structural failure.

2. The main bending failure mechanisms of the composite I-beam are the cracks propagate along the length direction of fibers at the top flange, the cracks propagate downward 
in the vertical direction, and the occurrence of cracks at the junction area between the top flange and web.

3. The top flange is the key area for the bending-induced damage of composite I-beams, and there is less visible damage on the web and bottom flange. When designing the composite I-beams to resist three-point bending loads, in addition to selecting the high-performance fibers and resin materials with excellent mechanical properties, the structural optimization of flanges should also be well considered.

\section{Acknowledgments}

The research is financially supported by The Open Project of the State Key Laboratory of Non-ferrous Metal Material Preparation and Processing (No.19AZO2) and the Opening Project of Zhejiang Key Laboratory of Clean Dyeing and Finishing Technology (No. QJRZ1907). We also acknowledge the support from the Fundamental Research Funds of Zhejiang Sci-Tech University (No. QJRZ1907), the Natural Science Foundation of Zhejiang Province(No. LGG21E050025) and the Open Project of Key Laboratory of Silk Engineering of Jiangsu Province(No. KJS2059).

\section{References}

[1] Garoushi, S. (2018). Dental composite materials for direct restorations. Fiber Reinforced Composites, 119-128.

[2] Huang, Y., King, D. R., Cui, W., Sun, T. L., Guo, H., et al. (2019). Superior fracture resistance of fiber reinforced polyampholyte hydrogels achieved by extraordinarily large energy-dissipative process zones. Journal of Materials Chemistry A, 7(22), 13431-13440.

[3] Rahman, A., Zakir, N., Abu-Mahfouz, I. (2018). Hybrid aluminum matrix composites (HAMCs) using powder metallurgy method. MS\&T18, 1304-1311.

[4] Priyanka, P., Dixit, A., Mali, H. S. (2019). High strength Kevlar fiber reinforced advanced textile composites. Iranian Polymer Journal, 28, 621-638.

[5] Galich, I. P., Viacheslav, S., Rudykh, S. (2018). Shear wave propagation in finitely deformed $3 D$ fiber-reinforced composites. International Journal of Solids \& Structures, 135, 303-304.

[6] Xu, J., Mi, S., Ming, W., An, Q., Chen, M. (2018). Research advances in cutting modeling of fiber reinforced polymer composites. Aeronautical Manufacturing Technology, 61(22), 16-23. (In Chinese)

[7] Benmokrane, B., Ali, A. H. (2018). Durability and long-term performance of fiber-reinforced polymer as a new civil engineering material. International Congress on Polymers in Concrete, 49-59.

[8] Aguilar, J., Winters, D., Sen, R., Mullins, G., Stokes, M.(2018). Fiber-reinforced polymer pile repair incorporating cathodic protection. Transportation Research Record, 2150(1), 111-118.

[9] Sajeeb, A. M., Babu, C. S., Arif, M. M. (2018). Evaluation of mechanical properties of natural fiber reinforced melamine urea formaldehyde (MUF) resin composites. Materials Today: Proceedings, 5(2), 6764-6769.
[10] Acosta-Enriquez, E. B., Pech-Canul, M. I., AcostaEnriquez, M. C. (2018). A revamped classification of composite materials. MA\&T18, 1319-1325.

[11] Condruz, M. R., Puscasu, C., Voicu, L. R., Vintila, I. S., Mirea, D. A. (2018). Fiber reinforced composite materials for proton radiation shielding. Materiale Plastice, 55(1), 5-8.

[12] Wang, Z., Zhao, X. L., Xian, G., Wu, G., Raman, R. K. S., Al-Saadi, S. (2018). Effect of sustained load and seawater and sea sand concrete environment on durability of basalt and glass-fibre reinforced polymer (b/gfrp) bars. Corrosion Science, 138, 200-218.

[13] Hung, P., Lau, K., Fox, B., Hameed, N., Lee, J., Hui, $D$. (2018). Surface modification of carbon fibre using graphene-related materials for multifunctional composites. Composites Part B Engineering, 133, 240-257.

[14] Liu, X., Wang, X., Xie, K., Wu, Z., Li, F. (2020). Bond behavior of basalt fiber reinforced polymer bars embedded in concrete under mono-tensile and cyclic loads. International Journal of Concrete Structures \& Materials, 14(1), 1-15.

[15] Shi, W., Han, D., Liu, Y., Hou, Y. (2019). Surface quality of aramid fiber composites with ultra-low temperature and micro-milling. China Mechanical Engineering, 30(9), 10561064. (In Chinese)

[16] Ma, D., Fang, Y., Wang, Q., Wang, Y., Xu, Q. (2018). Tension-compression fatigue behavior of high performance glass fiber reinforced resin matrix composites. Aerospace Materials \& Technology, 4, 63-66. (In Chinese)

[17] Hofstatter, T., Pedersen, D. B., Tosello, G., Hansen, H. N. (2017). Applications of fiber-reinforced polymers in additive manufacturing. Procedia Cirp, 66, 312-316.

[18] Asl, M. E., Niezrecki, C., Sherwood, J., Avitabile, P. (2017). Vibration prediction of thin-walled composite I-beams using scaled models. Thin-Walled Structures, 113, 151-161.

[19] Qin, X., Liu, H., Wu, C., Gu, Z. (2018). Semi-analytical solution of horizontally composite curved I-beam with partial slip. Steel and Composite Structures, 27(1), 1-12.

[20] Qin, X., Liu, H., Wu, C., Gu, Z. (2016). A trigonometric analytical solution of simply supported horizontally curved composite I-beam considering tangential slips. Mathematical Problems in Engineering, 2016, 1-12.

[21] Lacki, P., Derlatka, A., Winowiecka, J. (2019). Analysis of the composite I-beam reinforced with $P U$ foam with the addition of chopped glass fiber. Composite Structures, 218, 60-70.

[22] Naccache, F., El Fatmi, R. (2018). Buckling analysis of homogeneous or composite l-beams using a 1D refined beam theory built on Saint Venant's solution. Thin-Walled Structures, 127, 822-831.

[23] Alaedini, S., Kabir, M. Z., Al-Mahaidi, R. (2021). Stability performance of thin-walled pultruded beams with geometric web-flange junction imperfections. Journal of Building Engineering, 33.

[24] Shinokubo, M., Ohtani, A., Nakai, A., Hamada, H., Uozumi, T. (2010). Bending properties of fiber-hybrid i-shaped braided composite. Fiber, 66(11), 267-271.

[25] Zhu, P., Fan, H., Zhou, Y. (2016). Flexural behavior of aluminum $i$-beams strengthened by pre-stressed cfrp tendons. Construction \& Building Materials, 122(30), 607618. 